\title{
Error Analysis of PDR System Using Dual Foot-mounted IMU
}

\author{
Jae Hong Lee ${ }^{1}$, Hojin $\mathrm{Ju}^{2}$, and Chan Gook Park ${ }^{1,2^{*}}$ \\ ${ }^{1}$ Dept. of Mechanical \& Aerospace Engineering, Seoul National University, Seoul, 08826, Korea \\ ${ }^{2}$ Automation and Systems Research Institute, Seoul National University, Seoul, 08826, Korea
}

\begin{abstract}
In this paper, we analyze the position errors of the pedestrian dead reckoning (PDR) system using foot-mounted IMU attached to each foot, and implement PDR system using dual foot-mounted IMU to reduce the analyzed error. The PDR system using foot-mounted IMU is generally based on an inertial navigation system (INS). To reduce bias and white noise errors, INS is combined with zero velocity update (ZUPT), which assumes that the pedestrian shoe velocity is zero at the stance phase. Although ZUPT could compensate the velocity and position, the heading drift still occurs. When analyzing the characteristics of the position error, the error shows a symmetrical characteristic. In order to reduce this error, the previous researches compensate for both positions by applying feet position constraints. The algorithm consists of applying a conventional PDR system to each foot and fusion algorithm combining both. The PDR system using footmounted IMU, one on each foot, is based on integration approach separately. The positions of both feet should be in a circle with a radius as step length during walking. The designed filter is constrained so that the position of both feet are in a circular boundary. The heading error that is symmetrically drifted is corrected by the position constraint when the pedestrian moves straight. Experimental results show the performance and usability of each previous algorithm to compensate for symmetric heading errors.
\end{abstract}

\section{Introduction}

As interest in indoor location service increases, various studies have been conducted on the technique of estimating the position of a pedestrian in real time. Among them, the pedestrian navigation system using the inertial measurement (IMU) -mounted on the shoes is being actively studied because it provides the high accuracy position even with the inertial sensor without any device is installed in advance.

Pedestrian positioning techniques are classified as a method using the infrastructure and method that are not. The method with the infrastructure to estimate the pedestrian position uses $\mathrm{Wi}-\mathrm{Fi}, \mathrm{BLE}$ or IMUs instead of using an infrastructure. The pedestrian dead reckoning (PDR) system based on the IMU has an advantage in that it can estimate the pedestrian position without needing prior information [1-9]. Since MEMS-IMU is compact, it has been used in PDR systems. However, since the measurement performance of the MEMS-IMU is poor, various studies have been conducted to improve the estimation error of a PDR system caused by sensor error. One of the PDR system using the IMU is parametric approach, which estimates the pedestrian position by accumulating the step length and direction of movement. This method is mainly used when the sensor is attached to the waist or held by hand [1-6]. The other PDR system is integration approach, which consists of INS, which is applied when the sensor is attached to the shoe [7-9]. Because it is based on INS, there is a disadvantage that error of integration approach accumulates due to sensor measurement error. To reduce estimate error by measurement errors, the INS is combined with zerovelocity update (ZUPT) at the stance phase. Foxlin proposed PDR system using the extended Kalman filter (EKF) to estimate and subtract errors with ZUPT during the stance phase. This algorithm uses the assumption that the velocity is zero when the shoe stuck to the ground. This approach is known as ZUPT-aided INS or INS-EKFZUPT.

Although the bias and white noise errors of the sensor can be reduced through integration approach, it can also be caused by the unexpected gait characteristics [10]. The heading error cannot be calibrated in the method of integration approach. In this paper, IMUs are attached to both feet and PDR system is performed. We analyze the characteristics of the estimated position error using the experimental results. Based on the results of the analysis, we applied the previous research to compensate position error to the PDR system. Experiments show that the characteristics of the analyzed position error are compensated by the prvious algorithm.

\section{Methodology}

\subsection{Sensor}

We use the MTw from xsens for data acquisition. Table 1. Specification sensor mounted beside foot

Gyro Accelerometer

\footnotetext{
* Corresponding author: chanpark@snu.ac.kr
} 


\begin{tabular}{ccc}
\hline White noise & $0.05^{\circ} / \mathrm{sec} / \sqrt{\mathrm{Hz}}$ & $0.03 \mathrm{~g} / \sqrt{\mathrm{Hz}}$ \\
Turn-on bias & $20^{\circ} / \mathrm{hr}$ & - \\
\hline \hline
\end{tabular}

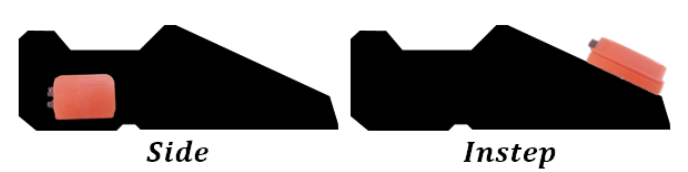

Fig 1. Location of sensor on shoe

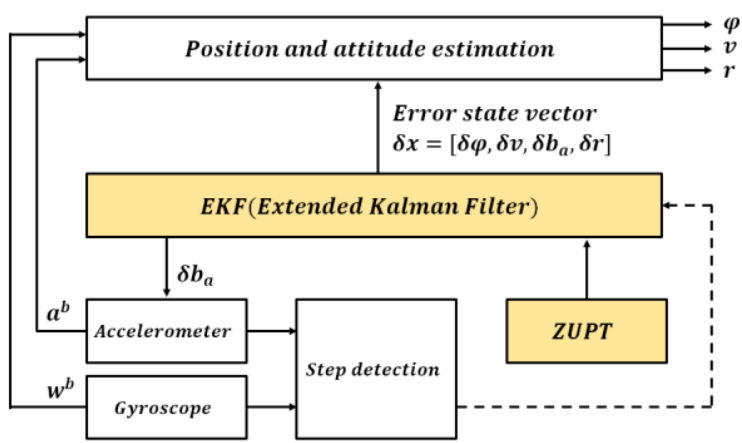

Fig 2. The PDR system with INS-EKF-ZUPT

The sensor specifications are summarized in Table 1. For the data analysis, the sampling rate of the sensor was set to the maximum value of $150 \mathrm{~Hz}$. The sensors are attached to both shoes, as shown in Fig. 1.

\subsection{Implementation of INS-EKF-ZUPT}

The PDR system uses a foot-mounted IMU based on INS to estimate pedestrian position. INS uses angular rate and acceleration measured from IMU to calculate attitude, velocity, and position. Unfortunately, the INS is diverging because of bias and white noise. In general, EKF and ZUPT (zero velocity update) are combined in order to solve this problem. Assuming that the speed is zero when the bottom of the shoe is stacked on the ground, the estimation error can be reduced through ZUPT. Whenever it is determined that the shoe is stacked to the ground, the EKF updates the velocity measurements using the ZUPT. The error state in a general PDR system consists of nine or 15 error states. The 15 error states include gyro and accelerometer bias, attitude, position, and velocity error. In this paper, we used 12 error states in the INS-EKFZUPT system. The error states are expressed as follows:

$$
\delta x=\left[\begin{array}{llll}
\delta \varphi, & \delta v, & \delta b_{a}, & \delta r
\end{array}\right]
$$

which contains the errors of attitude $(\delta \phi)$, velocity $(\delta v)$, acceleration bias $\left(\delta b_{a}\right)$, and position $(\delta r)$. The state transition matrix is

$$
\Phi=\left[\begin{array}{cccc}
I_{3 \times 3} & 0 & 0 & 0 \\
S \cdot d t & I_{3 \times 3} & C_{b}^{n} & 0 \\
0 & 0 & I_{3 \times 3} & 0 \\
0 & I_{3 \times 3} \cdot d t & 0 & I_{3 \times 3}
\end{array}\right]
$$

where $C_{b}^{n}$ is the rotation matrix that transforms values from the body (b) to the navigation (n) frame, $d t$ is the sampling time between two successive samples, and $S$ is the skew-symmetric matrix for accelerations in the navigation frame. The velocity error is used for the error measurement update in EKF. The error measurement is $\hat{v}-\left[\begin{array}{lll}0 & 0 & 0\end{array}\right], \hat{v}$ is the estimated velocity and $\left[\begin{array}{lll}0 & 0 & 0\end{array}\right]$ is the zero velocity, because the velocity of the foot is nearly zero during the stance phase. The measurement matrix is given as:

$$
H=\left[\begin{array}{llll}
0_{3} & I_{3} & 0_{3} & 0_{3}
\end{array}\right]
$$

The block diagram of the algorithm used for the test is shown in Fig. 2. Stance phase detection is required in order to use ZUPT. The stance phase is the moment when the bottom of the shoe is attached to the ground in step motion. We have modified the stance phase detection method using the magnitude and variation of acceleration and angular rate. The reason for subtracting the gyro bias error from the state is that there is no way to calibrate them using a filter.

\section{Error analysis about both feet}

Using INS-EKF-ZUPT can reduce errors caused by sensors. Experiments with sensors attached to both feet show the error characteristics of both shoes. Fig. 1 shows the position of the sensor attached to the shoe for the experiment. The first experiment attached the sensor to the side and the second experiment attached the sensor to the shoe.

Fig. 3 (a) shows the results of the first experiment. The pedestrian moved about 32 [m] straight. The heading angle for the first five steps was averaged to match the initial moving direction. The bias was removed through the initial alignment for about 10 [sec] to minimize the error caused by the gyro bias. Equation (4) is the result of attitude error estimation by gyro bias after alignment. $R W$ is the random walk of the gyro output, and $\sigma_{\text {align }}$ is the standard deviation of the bias removed from the initial alignment. When moving about 32 [m], an heading error of 0.0097 [deg] occurs. If this is converted into position error, it becomes $0.0059[\mathrm{~m}]$. Since the estimated position error due to the gyro bias is smaller than the error mean shown in Table 2, it can be seen that an element other than the sensor error exists.

$$
\delta \psi=\sigma_{\text {align }} \times t+R W \times \sqrt{t}=0.0097[\mathrm{deg}]
$$

Red is the estimated trajectory by the sensor mounted on the left shoe and blue is the estimated trajectory by the sensor mounted on the right shoe. The tendency of the position error is symmetrical with the sensors attached to each shoe. Table 2 shows the error mean and standard deviations of the final position by each shoe. The mean error due to left foot is negative and the error due to right foot is positive, so each error is symmetric. The same experiment was carried out by attaching a sensor on the shoe. Fig 3.(b) and Table 3 show the experimental results. Similar to when the sensor is attached to the side, the 
position error appears symmetrically depending on the right and left shoes in which the sensor is attached. Comparing two experimental results, It can be inferred that position error has a symmetrical tendency according to the left and right shoe attached to the sensor, but depending on the mounting position of the sensor, the direction of drift may be different.

\section{Applying dual foot-mounted PDR system}

There is a PDR system in which an IMU is attached to both shoes as a method of compensating the position error analyzed in Section 3. In order to compensate the position error by the heading drift that is symmetrical form, the position of both shoes can be bounded through the constraints of each other. There are several prior studies, in which the basic concept is to use a constraint that the relative position of the two shoes is less than the maximum step length of the pedestrian. In Fig. 4, when the right foot is stepped, the estimated position due to heading drift exceeds the maximum step length $(\gamma)$. At this time, it is possible to prevent the position error from diverging due to the heading drift by correcting the estimated position into the maximum step length [11-14].

\subsection{Single foot-mounted IMU}

In each foot, a PDR system is constructed by the INSEKF-ZUPT method. Detailed algorithms are described in section 2. The PDR system operates independently on each foot.

\subsection{Dual foot-mounted IMU}

The estimated positions in each PDR system are combined in the stance phase. As shown in Fig. 4, when the left foot is in the stance phase and the right is in the swing phase, the position of the right foot is corrected. The conditions for correction are as follows:

$$
d_{k}=\operatorname{norm}\left(\hat{p}_{k}^{l}-\hat{p}_{k}^{r}\right)>\gamma
$$

$d_{k}$ is the relative position of both feet, $\hat{p}_{k}^{i}$ is the estimated position of the $i \in\{l e f t$, right $\}$ side foot.

When the above condition is satisfied, the filter is updated with the position of the right foot considering the constraint.

One way to determine the measurements is to correct the position with the maximum step length (Method 1). The measurement is determined by Eq. (6). This method was proposed by G. V. Prateek [11, 12].

$$
z_{k}^{i}=\frac{2}{d_{k}}\left(\left(d_{k}-\gamma\right) \hat{p}_{k}^{j}+\gamma \hat{p}_{k}^{i}\right)
$$

\section{$i, j \in\{l e f t$,right $\}$ and $i \neq j$ is satisfied.}

The second method is to determine a measure that meets the minimum value of the cost function (Method 2). The measured value satisfying this is expressed as Eq. (8) This method was proposed by R. Girisha $[13,14]$.

$$
\begin{aligned}
& J=\left\|\hat{p}_{k}^{i}-z_{k}^{i}\right\|_{2}^{2}+\lambda\left(\left\|\hat{p}_{k}^{i}-\hat{p}_{k}^{j}\right\|_{2}^{2}-\gamma^{2}\right) \\
& z_{k}^{i}=\frac{1}{2 d_{k}}\left(\left(d_{k}+\gamma\right) \hat{p}_{k}^{i}+\left(d_{k}-\gamma\right) \hat{p}_{k}^{j}\right)
\end{aligned}
$$

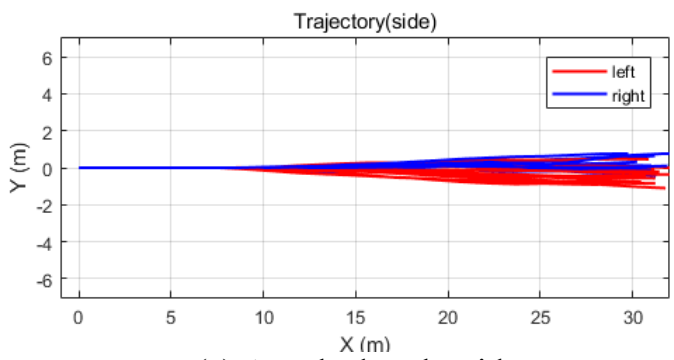

(a) Attached to the side

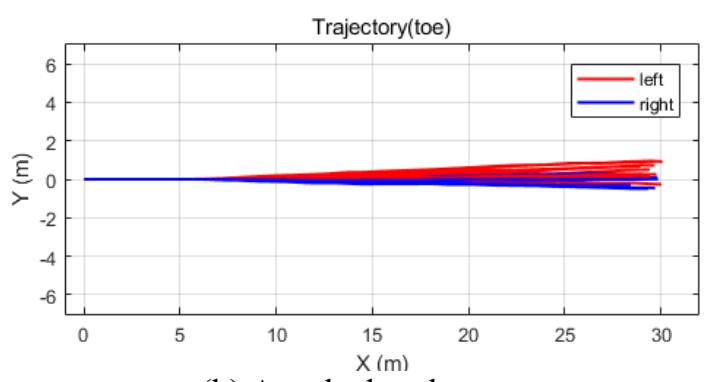

(b) Attached to the top

Fig 3. The estimated trajectory

Table 2. Y-axis position error (side)

\begin{tabular}{ccc}
\hline \hline & Mean $[\mathrm{m}]$ & $\sigma[\mathrm{m}]$ \\
\hline Left & -0.4157 & 0.5484 \\
Right & 0.1688 & 0.3874 \\
\hline \hline
\end{tabular}

Table 3. Y-axis position error (top)

\begin{tabular}{ccc}
\hline \hline & Mean $[\mathrm{m}]$ & $\sigma[\mathrm{m}]$ \\
\hline Left & 0.4411 & 0.3804 \\
Right & -0.0419 & 0.2675 \\
\hline \hline
\end{tabular}

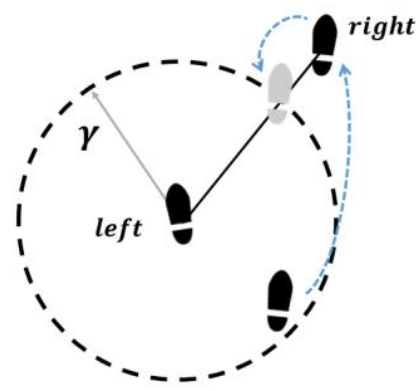

Fig 4. Movement of each foot and maximum step length

We define measurement matrix $H$ as follows:

$$
H=\left[\begin{array}{llll}
0_{3} & 0_{3} & 0_{3} & I_{3}
\end{array}\right]
$$


The algorithm of the previous study was applied to the data analyzed in Section 3. Table 4 shows the error mean for the end positions. It can be confirmed that the mean of the position error estimated from both feet becomes smaller when using the method 1 and 2. Fig. 4 shows the results of one experiment. The $y$-axis position is diverged, but dual foot-mounted IMU algorithm with method 1 and 2 make y-axis position is bounded.

However, fig. 4. (b) shows the limitation of the previous study. The position of both feet is compensated to midway through the constraint. If the symmetrical position error of both feet is not similar, and the degree of divergence of one foot is steeper, The position corrected by the filter is biased in the direction of the foot that is steeply diverging. Since the positional error of the left foot was more biased than the right foot, the corrected position was also biased downward without estimating it as a straight line (Fig 4.(b))

Table 4. Y-axis position error mean for each method

\begin{tabular}{ccc}
\hline \hline & Left & Right \\
\hline None & $-0.4157[\mathrm{~m}]$ & $0.1688[\mathrm{~m}]$ \\
Method 1 & $-0.3414[\mathrm{~m}]$ & $0.0713[\mathrm{~m}]$ \\
Method 2 & $-0.3505[\mathrm{~m}]$ & $0.0952[\mathrm{~m}]$ \\
\hline \hline
\end{tabular}

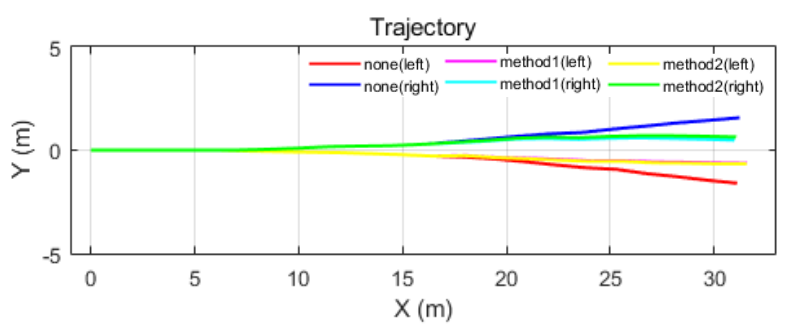

(a) Case 1

Trajectory

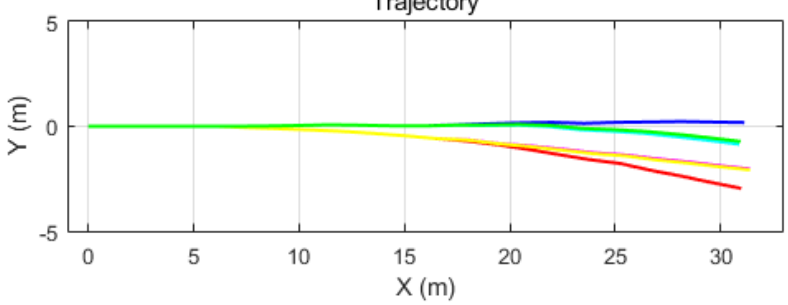

(b) Case 2

Fig 4. The estimated trajectory with each method

\section{Conclusion}

The purpose of this paper is to analyze the tendency of heading drift and to apply previous studies to reduce estimation error due to drift. To analyze the heading drift, IMU were attached to both feet and we collected data through several experiments. The analysis showed that both side heading drifts were symmetrical regardless of sensor location. To compensate for symmetric heading drift, previous studies use a constraint that the relative distance of the estimated position of each foot cannot be greater than the maximum step length. By using these constraints to compensate for position in the filter, the position is bounded. We confirmed that the method applied through experiment was valid and shown the limitation of previous studies

This work was supported by Institute for Information \& communications Technology Promotion(IITP) grant funded by the Korea government(MSIT)(No. 2018-0-00781, Development of human enhancement fire helmet and fire suppression support system).

\section{References}

1. E. Foxlin, IEEE Comput. Graph, 25, (2005)

2. S. Godha, G. Lachapelle, Meas. Sci. Technol., 19 (2008)

3. R. F. Alonso, E. Z. Casanova, J. G. García-Bermejo, J. Physical Agents, 35-43 (2009)

4. A. R. Jiménez, F. Seco, J. C. Prieto, J. Guevara, 2010 7th Workshop on Positioning, Navigation and Communication, 135-143 (2010)

5. M. S. Lee, C. Park, C. W. Shim, Proc. 2012 Int. Meet. Inst. Navig, 922-927 (2012)

6. H. Ju, et al., Meas. Sci. Technol., 27, (2015)

7. S. Y. Cho, and C. G. Park, J. Navig., 59, 135-153 (2006)

8. J. Kappi, S. Jari, and S. Jukka, Proc. of the 14th Int. Technical Meeting of the Satellite Division of the Institute of Navigation, 1369-1373 (2001)

9. B. Zhou, Q. Li, Q. Mao, W. Tu, and X. Zhang, IEEE Trans. Hum. Mach. Syst, 45, 562-574 (2015)

10. J. Nilsson, S. Isaac, and P. Händelm, 2013 Int. Conf. on Indoor Positioning and Indoor Navigation, (2013)

11. J. O. Nilsson, et al., EURASIP J. Adv. Signal Processing, 164 (2013)

12. G. V. Prateek, R. Girisha, K. V. S. Hari, and P. Händel, in Proceedings - International Conference on Intelligent Systems, Modelling and Simulation, 208-213 (2013)

13. R. Girisha, G. V. Prateek, K. V. S. Hari, and P. Handel, 2014 International Conference on Signal Processing and Communications (SPCOM), 1-6 (2014)

14. W. Shi, Y. Wang, and Y. Wu, Sensors, 17 (2017) 